\title{
Sobre el lugar donde pensar: filosofía y universidad moderna
}

\author{
The place for thinking: \\ Philosophy and the Modern University
}

\author{
Juan Andrés Queijo Olano \\ Facultad de Información y \\ Comunicación \\ Universidad de la República \\ juan.queijo@fic.edu.uy
}

Recebido em 14/09/2017

Aceito em 02/10/2017

\begin{abstract}
Resumen
El presente trabajo intenta brindar una línea de respuesta histórica, disciplinaria y tentativa para la pregunta sobre por qué las universidades constituyen espacios de conocimiento que, pese a su reconocida importancia, se mantienen alejadas de las sociedades que la contienen. Se trata de una respuesta histórica en dos sentidos. En primer lugar, porque ubica su respuesta en un acontecer histórico concreto, este es, el surgimiento de un nuevo tipo de universidad en el siglo XIX europeo. Es también una respuesta disciplinaria porque, sensu strictu, la pregunta por las universidades es una pregunta por las disciplinas que la conformaron. Finalmente, es una respuesta tentativa. Es una invitación a poder pensar este complejo proceso de construcción de las universidades contemporáneas a partir de algunos trozos de la gran Historia de la Ciencia y la Filosofía Occidental. Mirar los lugares donde se ha pensado, donde el conocimiento se ha provocado, es simplemente una de las formas para entender el proceso social más amplio y profundo, que ha llevado a las universidades a ubicarse en ese lugar donde están actualmente.
\end{abstract}

Palabras clave: Universidad Moderna, Filosofía alemana, Educación.

\section{Abstract}

This work aims at presenting a historical, subject-oriented and tentative answer for the question: why do universities, as particular knowledge-places, and besides their well-known importance, are still isolated from societies? The first answer is developed from a micro-historical perspective, focused on the academic trail of relevant nineteenth century European philosophers who discussed the role of philosophy and science in the modern university. It is also a subject-oriented perspective because it intends to acknowledge how philosophers and scientists played their arguments in favor of the development of their fields and against external power. Finally, it is a tentative answer. In fact, it is an invitation to think the complex process of contemporary universities, from the perspective of the Western History of Science and Philosophy. It is my belief that analyzing the agents and places where scientific and philosophical knowledge has been produced, allows understanding some aspects of the wide and deep process that leads to the current situation of our universities.

Keywords: Modern university, German philosophy, Education. 


\section{Introducción}

¿Por qué las universidades mantienen una lejanía con la sociedad y qué rol juega el conocimiento en esa separación? Aunque de forma demasiado general, la pregunta resume y señala el punto central de este trabajo. Antes de adentrarnos en las respuestas que pueden ayudar a explicar este fenómeno, vale la pena preguntarse sobre la legitimidad de la afirmación contenida entre los signos de interrogación. ¿Es cierto que las universidades se mantienen alejadas de la sociedad? ¿Es cierto que funcionan como Torres de Marfil?

Una forma de dar respuesta a estas últimas preguntas es a través de la Historia. En ese sentido, una primera parte de este trabajo intentará acercar reflexiones sobre la universidad cuando ésta se refunda bajo el signo de la modernidad, en la Alemania de inicios del siglo XIX. El carácter paradigmático de esta universidad parece ser reconocido casi unánimemente en los estudios sobre las universidades. Se analizarán los escritos de autores que constituyen parte de la literatura clásica de lo que se suele conocer bajo el rótulo de la Teoría de la Universidad (Bonnevecchio, 1995; Fuller, 2009; Gordon, 2005; Grant, 1996). El porqué de esos textos o esos autores no tiene una explicación clara, más que la de corresponder a escritos que reflexionan sobre la condición de la Universidad moderna, y en tal sentido no pretenden mostrarse como el examen exhaustivo de toda una época. Por ello, tampoco, lo que pueda concluirse de ellos no aspira a ser más que un aporte a una reflexión sobre la condición de la universidad. Así, este recorrido comenzará con el clásico texto del cardenal inglés John Henry Newman, La idea de una universidad, de 1852; siguiendo con Immanuel Kant y El Conflicto de las Facultades; la disputa entre Hegel y Schopenhauer sobre el rol de la filosofía y las universidades; para finalizar con los pensamiento de Friedrich Nietzsche sobre esa institución. El simple contrapunto de estos filósofos nos permitirá mostrar algunos elementos de reflexión general sobre la naturaleza de ese aislamiento.

Un segundo aspecto que merece aclaraciones previas es el de las disciplinas que serán nombradas en este trabajo. El problema de las universidades es -entre otros- el problema de sus disciplinas, y aquí nos referiremos especialmente a dos: la Filosofía y la Ciencia. En los autores que veremos, se pueden ver alternadas e intercaladas las preocupaciones por la institución universitaria en tanto problemas de la Filosofía, y viceversa. Este uso indiscriminado y entremezclado entre Filosofía, Universidad y Ciencia, nos habla del período sobre el cual estamos reflexionando. En primer lugar, la Universidad del siglo XIX se ocupa de pocas disciplinas. Arrastrando y manteniendo firmemente todavía la estructura medieval, esta institución se reducía a la formación en Teología, Medicina y Leyes, a las que podían sumarse las llamadas Artes Liberales (trivium). La inclusión del estudio de las "ciencias" en el seno de la Universidad es uno de los hitos que se le adjudicó a la Universidad de Berlín, y en especial a Wilhelm Von Humboldt. Estas "ciencias" se presentaban bajo el rótulo de una Filosofía de la Naturaleza, por eso deben tenerse recaudos cuando se interpretan los términos en cuestión anacrónicamente. Obviamente, esta situación cambiaba según las universidades que desde el medioevo fueron marcando también sus propios estilos y formas de especialización. Así, se reconoce en Oxford una tradición en matemáticas y ciencias físicas mucho más acentuada que en la Universidad de Paris, cuya formación estaba casi exclusivamente abocada a Aristóteles y sus comentadores (Gilson, 1989).

De forma general, vale aclarar que este texto no tiene un afán conclusivo ni definitivo. Es una invitación a pensar los problemas de la universidad, la ciencia universitaria y su llamada autonomía, que hoy se nos presentan en el siglo XXI, tomando como insumo las reflexiones de los pensadores de la época en la cual la universidad tomaba un nuevo rumbo y forma hacia su expresión moderna. Intentar reconocer algunos de los problemas que recorrieron toda una época donde las universidades refundaban su camino, espera ser uno de los aportes que puedan servir para entender algunos de los problemas que atraviesa la universidad contemporánea.

\section{John Henry Newman}

El cardenal John Henry Newman (1801-1890) fue una figura sumamente influyente en su época para la construcción de una nueva universidad. En el año 1852, habiendo abdicado al anglicanismo y habiéndose declarado católico, es llamado por el obispo de Dublín a fundar una universidad católica en esa ciudad. Newman imparte en ese año una serie de lecciones que no solo dan muestra del rigor intelectual por el que era reconocido en esa época, sino que además provoca la redacción de un escrito que será constantemente 
referenciado desde ese momento. The Idea of a University es un trabajo extenso, complejo y altamente pertinente en lo que refiere a la construcción de la institucionalidad universitaria en el siglo donde las condiciones de producción se vieron revolucionadas por la forma industrial.

De hecho, parece ser este uno de los principales asuntos de preocupación para Newman: el valor del conocimiento ante el advenimiento de una lógica utilitaria que parecía devorar todas las dimensiones de la vida social británica. Este gran tema se expresa al menos de tres formas diferentes a lo largo de su obra: i) como justificación del conocimiento por el conocimiento mismo; ii) el papel de las universidades en la encrucijada del conocimiento liberal y el conocimiento utilitario; y iii) la enseñanza del conocimiento y la creación de una clase social: los gentlemen.

Este tipo de análisis nos da muestras, en el fondo, del tipo de lugar que ocupará la Universidad en el proyecto que Newman pretendía desarrollar en Dublín, y sobre todo, el lugar dentro de la sociedad por el cual la ciencia puede encontrar su mejor desarrollo.

Una de las primeras preocupaciones de Newman fue defender la importancia del conocimiento por el conocimiento per se. Como fue dicho, en el contexto de avanzada del capitalismo y de los procesos de industrialización donde el conocimiento aplicado a la producción parece imponerse como norma, el cardenal Newman pretende que las Universidades no pierdan esa condición "natural" por la cual el conocer tiene que mantenerse ligado a esa curiosidad del hombre por el mundo.

Mi razón para tratar esta cuestión [el fin de la enseñanza universitaria] es porque ella ocupa un lugar muy particular en mi corazón. Pero además me refiero a ella porque ha sido objeto de grandes controversias, cuando después de un profundo debate quedó en evidencia que durante casi un siglo la universidad ya no educaba a sus jóvenes con propiedad, por lo que se vio obligada a reflexionar sobre sus responsabilidades. Los argumentos en contra de los estudios universitarios hacían referencia a su lejanía con las ocupaciones y los deberes de la vida, es decir, a su inutilidad (Newman, 2016: p. 22).

Newman reconoce los argumentos que en su tiempo se daban contra la formación que las universidades brindaban a sus jóvenes: la supuesta inutilidad del conocimiento filosófico o científico. Ante esta afrenta, el clérigo asume la tarea de fundamentar la universidad en razón de justificar el cultivo de un tipo particular de conocimiento. "Una universidad, en su esencia más pura, tiene por objeto y misión la cultura intelectual. Esto significa ejercitar la mente y educar al intelecto para razonar bien en todas las cosas, para llegar a la verdad y para comprenderla." [Newman, 2016: p. 102]

El reconocimiento de esta función para las universidades implica la distinción de, al menos, dos formas de concebir el conocimiento. Dos formas que como veremos, trazada esa distinción, sirven también como forma de distinción social. Estas dos formas dividen al conocimiento liberal, del conocimiento utilitario.

El término "liberal" aplicado al conocimiento y a la educación, alude a una idea específica que trasciende en el tiempo (...) Me refiero al conocimiento liberal, que es específicamente el que llamo filosofía o, en un sentido más amplio de la palabra, ciencia. La enseñanza de este conocimiento es el ámbito de la universidad, e independiente de lo que se diga, para que el conocimiento sea considerado en su sentido más pleno, no puede ser apreciado según una visión popular, sino más trascendente, como una filosofía. (Newman, 2016: p. 92-93).

Esta clasificación aristotélica determina que por detrás de ciertos conocimientos visibles, que aplican a la realidad material, se esconden otros, de carácter más esencial y, por lo tanto, superior al anterior. Lo interesante de este texto de Newman es que, -y es este precisamente el segundo punto que merece un especial tratamiento-, él ve claramente en el problema de la utilidad del conocimiento, de la utilidad de la ciencia, el escollo ante el cual se enfrentarán las universidades desde ese momento. 
La universidad debe elegir qué proyecto tomar: el de la búsqueda del conocimiento; el de la formación profesional o el de la formación religiosa.

“Es la adquisición y el logro del conocimiento responsabilidad de la educación universitaria? ¿O más bien está su cultivo dirigido a la experiencia de algunas profesiones y ocupaciones particulares? ¿o se puede decir que el mismo conduce a conductas morales y religiosas?” (Newman, 2016: p. 103)

Más interesante que la respuesta que Newman pueda ofrecer a esta serie de preguntas, es cómo aparece desde ya resumido el problema central sobre la condición del conocimiento producido en las universidades. La tensión entre brindar un conocimiento que posibilite utilidades prácticas al individuo, entre aquel que lo eduque en su erudición intelectual y, finalmente, aquel que lo eduque en sus convicciones morales. Las universidades atravesarán -desde entonces- esta misma encrucijada político-filosófica concerniente a su condición y que se regula, directamente, con el tipo de conocimiento que parece poder producir.

Finalmente, a esta relación entre tipos de conocimiento y fines de la Universidad, deberemos agregar un elemento que complejiza y supone otra forma de entender el aislamiento de la universidad en las sociedades. Si como sugiere Newman los propósitos de la universidad deberían estar orientados a la "cultura intelectual", y no a lo que denomina "la filosofía de lo útil" - que "tiende a aumentar la comodidad tanto física como social"-, estos propósitos serán también el fundamento de aquella clase que lleve adelante el modelo de universidad iluminista, fundado por y para la Filosofía.

"La filosofía, por muy profunda y esclarecedora que sea, no proporciona el control sobre las pasiones ni dirige nuestros principios; la educación liberal no hace ni al cristiano ni al católico, sino que al gentleman. Este es el objeto de una universidad; formar una persona correcta, con claridad de mente, noble, culta y de buen gusto. Todas estas cualidades son connaturales al cultivo del conocimiento (...)" (Newman, 2016: p. 97)

Esta consideración de Newman muestra cómo ciertas variables sociológicas también se introducen en la forma en que se definen las universidades y el tipo de conocimiento que ellas producen. No es meramente a partir del tipo de conocimiento que se desarrolla un proyecto educativo: será la clase que allí se conforme la que transfiera sus valores ("claridad de mente", "noble", "culta", "de buen gusto") a la forma institucional que se va delineando. En otras palabras, no son meramente los elementos epsitemológicos que definen el conocimiento los que determinan el tipo de entidad universitaria a crearse; con ellos se introducen otro tipo de valoraciones de carácter moral, estético, político, social, que ayudan a demarcar la identidad institucional de las universidades.

\section{Immanuel kant}

Comienza Kant su sugerente escrito sobre El conflicto de las Facultades de la siguiente forma.

No tuvo mala ocurrencia aquel que concibió por primera vez la idea y propuso que la misma se llevara a cabo públicamente, de tratar, por así decirlo, industrialmente todo el conjunto de la ciencia (lo harían las cabezas que se dedican a ella) dividiendo el trabajo; se nombrarían tanto maestros públicos o profesores como materias científicas, y convertidos en sus depositarios construirían juntos una especie de institución erudita llamada Universidad (o Escuela Superior) autónoma (...) (Kant, 2004: p. 19)

Probablemente estemos ante uno de los primeros textos que evidencia la condición industrial del conocimiento por su órgano productor, la Universidad ${ }^{1}$. ¿Qué es la Universidad sino esa institución que valida y promueve la división del trabajo intelectual en formas arbitrarias? En tiempos de Kant esta división del trabajo se establecía entre Facultades Superiores (Teología, Derecho y Medicina) y una Facultad Inferior (la Filosofía); pero esta estrategia de clasificación, denuncia Kant, solo corresponde a la visión de quien nada entiende de cuestiones del saber: el gobierno. El interés de este, según Kant, está en impresionar a su pueblo "por medio de ciertas doctrinas", asegurándole a los individuos "el bien eterno de cada

$1 \quad$ El texto de Kant es de 1798. 
uno, después el bien civil, como miembro de la sociedad, finalmente el bien corporal (vida larga y salud)" (Kant, 2004: p. 26)

Entre las Facultades Superiores, si nos ubicamos desde la Facultad de Teología hacia la de Medicina, podemos ver el camino hacia la autonomía propia de las ciencias, en un sentido muy peculiar. Kant nos muestra cuáles son las condiciones de una posible participación del gobierno en los conocimientos de las Facultades. Y esas condiciones, en este caso, tienen que ver con la articulación de los conocimientos alrededor de un códice, un código de normas que guían el campo de saberes. Es evidente que en la Teología ese código no es otro que La Biblia, así como en el Derecho ese código son las leyes que constituyen las normas de convivencia ciudadana. Pero a medida que nos acercamos a las ciencias, es clara la forma en que esos códices van perdiendo su carácter dogmático e instructor, y es la racionalidad la que comienza a operar en su lugar. En otras palabras, anacrónicas al contexto pero clarificantes para nuestros intereses, podemos decir que las disciplinas profesionales basan su autonomía en un código que es maracado socialmente; mientras que las disciplinas académicas prescinden de un aparato instructor tan fuerte: a ellas las guía la razón, que solo se valida en el corazón mismo de la comunidad de especialistas. La Medicina parece mostrar esa doble condición, según Kant, y es importante ver cómo, de cierta forma, emerge en su discurso la idea de "libertad" asociada a una Facultad:

Esta Facultad [la de Medicina] es entonces mucho más libre que las dos primeras entre las Facultades superiores y está estrechamente vinculada a la Facultad de Filosofía; pero en lo que se refiere a las enseñanzas destinadas a formar a los médicos es totalmente libre, porque para ellas no existen libros sancionados por una autoridad suprema, sino libros inspirados en la naturaleza; tampoco hay leyes en un sentido propio (si se comprende por ellas la voluntad inflexible de un legislador), sino solo reglas (edictos); conocerlas no es ninguna ciencia; una ciencia consiste en un complejo sistemático de teorías, que la Facultad posee sin duda, pero que el Gobierno no tiene la competencia de sancionar (porque no figura en ningún código) (Kant, 2004: p. 33-34).

Si algo determina la posibilidad de aislamiento de un saber, no es otra cosa para Kant que la posibilidad de fundar esos conocimientos en la legislación de la razón. De cierta forma, el carácter de inferioridad de la Filosofía (el cual, obviamente, Kant no comparte) se encuentra en estrecha relación con la imposibilidad del gobierno de acceder a sus discusiones y fundamentos. No es extraño entonces que a partir de Kant, quien reformula la forma de hacer y entender la Filosofía, la utilidad comience hacer una variable para juzgar este tipo de conocimientos, que mediante un lenguaje cada vez más especializado comienza a alejarse del alcance social

Se puede llamar Facultad inferior a aquel curso de la Universidad que solo se ocupa, o en la medida que solo se ocupa, de doctrinas que no son aceptadas como normas por orden de un superior [...] Al poder de juzgar de un modo autónomo, es decir, libremente (según los principios de la inteligencia en general) se le llama razón. Y por ello debe considerarse a la Facultad de Filosofía, por el hecho de tener que responder de la verdad de las doctrinas que debe aceptar o simplemente incluir, libre y dependiente de la legislación de la razón, y no de la del gobierno.

En una Universidad, pues, debe instituirse un departamento tal, es decir, tiene que funcionar una Facultad de Filosofía. [Kant, 2004: p. 34-35].

Los pasajes anteriores de Kant permiten vislumbrar la forma moderna de justificación de autonomía de las Universidades a través de la ciencia. Los saberes que dependen de la razón no deben inmiscuirse con aquellos orientados a la población, son saberes que solo reconocen su autoridad en tanto no se los someta al juicio público. La Facultad Inferior de la Filosofía, en la cual residen los saberes de la ciencia histórica y de las ciencias racionales puras, no debe ser restringida en su libertad.

Nuevamente, la expresión aislacionista, autónoma, libre de la Universidad adquiere sentido solo si es definida negativamente respecto a la injerencia de un poder de gobierno que la oprime. Kant, como antes Platón, justifican la posición de aislamiento filosófico como un resguardo de la razón ante los intereses cotidianos de 
la población y su gobierno. Una vez más, la Filosofía (ahora como el conjunto de saberes humanos) reclama su lugar, su espacio en la Universidad, pero exige de ésta protección para no sufrir los embates del mundo.

\section{Arthur Schopenhaver}

Una discusión históricamente relevante sobre el lugar de la Filosofía en las universidades se da en forma de controversia entre dos grandes filósofos que se disputaron (o uno quiso disputarle al otro), la supremacía intelectual en el seno de la Universidad de Berlin en los albores de su creación.

Las controversias -algunas más cotidianas y domésticas que otras- entre Schopenhauer y Hegel tomaron lugar cuando el primero vio resumida la tarea de la filosofía académica bajo el paraguas conceptual que había abierto Hegel y que, casi sin excepciones, había dejado a toda otra forma de pensamiento filosófico a la intemperie. Y si bien estas discusiones "académicas" se dan cuando, precisamente, la tarea filosófica comienza su profesionalización en los ámbitos universitarios, lo que resulta interesante para nuestros propósitos es ver cómo, detrás de las cuestiones más mundanas, residen también ideas sobre cómo el pensar dentro de las universidades es una forma de rechazo a filosofías alternativas, como en ese entonces se presentaba la filosofía de Schopenhauer.

Es en el verano de 1811 cuando Arthur Schopenhauer ingresa en la Universidad de Berlín, con la idea firme de conocer a quien en ese entonces se había erigido como la referencia filosófica de Alemania, en tanto era considerado como el sucesor más atendible y respetado de Kant: Johann Gottlieb Fichte. Que una universidad de tan solo un año de existencia pudiera contar entre sus docentes a las principales personalidades filosóficas de la época, solo puede ser explicado si entendemos el papel que la Universidad de Berlín, la Humboldt Universität, cumplió en ese período en el desarrollo de Alemania.

Tras la catastrófica derrota de Prusia contra Napoleón en 1806, y tras la pérdida de la vieja Universidad de Halle, que fue consecuencia de aquella derrota, los reformadores prusianos proyectaron un nuevo tipo de educación que debían recibir los cerebros para renovar las instancias del Estado. Habían conseguido que Wilhelm Von Humboldt se sumase al plan de fundar una nueva universidad, la cual, según palabras del rey, «debía reemplazar con fuerzas espirituales las fuerzas físicas que el Estado había perdido». En un primer momento, permitieron que Humboldt llevase a la práctica sus elevadas ideas sobre una educación humanística que debía ser algo más que mera preparación profesional. Se dio un peso especial a las «humanidades», es decir, a las disciplinas filosóficas, filológicas y teológicas. Se quería reunir en Berlín a las mejores cabezas en tales disciplinas. Con Fichte, Schleiermacher y Wolf, el propósito quedó cumplido. El primero de estos fue elegido rector pocos días antes de la llegada de Arthur [Schopenhauer] (Safranski, 2004: p. 167).

La Universidad de Berlín se vuelve, en su creación, una institución respaldada y creada para reemplazar "las fuerzas espirituales" de la nación en construcción. En este sentido, el rechazo que se genera en Schopenhauer contra la figura de Hegel resulta de cómo este último representa el papel de la filosofía oficial, o más aún, cómo Hegel avala y fundamenta la posibilidad de una filosofía oficial. Para Schopenhauer esta posición aleja a la filosofía de su lugar originario, en el sentido de que -cita a Platón para dar cuenta de esto- debe ser "cultivada con dignidad" por "hombres rectos". Así, el desarrollo de la filosofía en la Universidad alemana de comienzos del siglo XIX, lejos de brindarle un marco para su desarrollo libre y autónomo -como parece ser que el espíritu de la Modernidad promete para sus instituciones-, devuelve a la universidad una serie de restricciones y limitaciones que se conforman sobre las nuevas dinámicas académicas que se establecen en el relacionamiento laboral y social de la institución. Un ejemplo de cómo Schopenhauer quiso combatir esta situación fue colocar sus cursos, una vez que accedió al cargo de docente en la Universidad de Berlín, en los mismos días y horas en que Hegel daba los suyos². Si bien el resultado le fue bastante adverso, nos da precisa

2 Todavía en Dresde, y antes de haber culminado su habilitación, pretende que el decano de la facultad berlinesa anuncie ya en el repertorio de lecciones de la universidad el curso que impartirá. Quiere enseñar acerca de «toda la filosofía, es decir, la doctrina de la esencia del mundo y del espíritu humano». Y, por lo que se refiere a las horas para impartir en clase, considera que «las más convenientes serían [...] las mismas en las que el señor profesor Hegel da su curso principal»". [SAFRANSKI: 2004, p. 334]. 
referencia de la estrategia política de Schopenhauer: presentar alternativas a la filosofía oficial de Hegel (lo que llamaba "filosofía de cátedra"), para salvar a la filosofía de su propia burocratización.

(...) He llegado poco a poco a la opinión de que la mencionada ventaja de la filosofía de cátedra es superada por el prejuicio que ocasiona la filosofía como profesión a la filosofía como libre investigación de la verdad, o la filosofía por encargo del gobierno a la filosofía por encargo de la naturaleza y la humanidad (Schopenhauer, 2009: p. 167)

Lo que resulta relevante para nuestros propósitos es mostrar cómo la discusión sobre el lugar de la Filosofía, ese lugar para el pensamiento sin restricciones, se vuelve una discusión sobre las consecuencias de la profesionalización académica. Lo que veremos latente en las ideas de Schopenhauer es que, la persistencia de las dinámicas sociales en el nuevo proyecto de Universidad llevarán a la solidificación de ciertas relaciones de poder, y concretamente en el contexto alemán, a la creación de una nueva clase social: la clase de los académicos. Al igual que Newman reconoce la conformación de esta clase social en la universidad irlandesa de mediados de siglo XIX, los gentlemen; en el caso alemán será la clase de los german mandarines ${ }^{3}$ quienes irán edificándose como grupo social al tiempo que el proyecto de universidad se desarrolla modernizándose. Esta clase académica encuentra su verdadero celo:

en conseguir con honor unos honrados ingresos para ellos junto con sus mujeres e hijos, y también gozar de un cierto prestigio ante la gente ; en cambio, el ánimo que se agita en lo profundo del verdadero filósofo, cuyo total y enorme celo se halla en buscar una explicación de nuestra existencia, tan enigmática como penosa, es contado por ellos entre los seres mitológicos(...) es sumamente infrecuente que un auténtico filósofo haya sido a la vez un docente de la filosofía. (Schopenhauer, 2009: p. 168-169)

El desafío ante el cual nos coloca Schopenhauer está en indagar sobre la filosofía en las instituciones, en la universidad. ¿Puede haber una actividad filosófica plena -en su sentido más profundo, intelectual, espiritualsi esa actividad es desarrollada profesionalmente? Schopenhauer nos invita a dudar de esta posibilidad, no únicamente porque a la manera socrática se considere que no deben recibirse ingresos por enseñar filosofía (Schopenhauer acusa a Fichte de sofista, y más adelante a Hegel de pseudofilosofía), sino porque hacerlo implica envolverse en una serie de contratos normativos que recaen en la actividad, y que no son otra cosa que las reglas institucionales impuestas en las universidades.

En otras palabras, podríamos decir que el proyecto kantiano de mantener salvaguardado el pensamiento y la autonomía de ideas en las Universidades, a través de la Filosofía (y la razón), no consiguió mantener esa pureza ante la presión de la institución. La Filosofía, nos dice Schopenhauer, no ha podido aislarse del resto de las influencias que someten al conocimiento, y esto es tanto por una incapacidad de los filósofos profesionales, "la filosofía de cátedra", como de la propia naturaleza filosófica, que no admite dos posibilidades.

La filosofía es, por su naturaleza, exclusiva: fundamenta la forma de pensar de la época: por eso el sistema dominante no soporta otro junto a sí (...) Como consecuencia de todo esto, quien no aspire a una filosofía de Estado y una filosofía de broma sino al conocimiento y, por tanto, a la búsqueda de la verdad tomada en serio y sin consideraciones, tendrá que buscarla en cualquier parte antes que en las universidades, donde su hermana, la filosofía ad normal conventionis, ejerce el mando y escribe el menú. [Schopenhauer, 2009: p. 183]

\section{FRIEDRICH NIETZSCHE}

El último filósofo que veremos atacar virulentamente todo el espectro burocrático que se fue conformando, a medida que el proyecto de universidad moderna se desarrollaba en Alemania, fue Friedrich Nietzsche. Nietzsche es, sin lugar a dudas, un filósofo que ha actuado y filosofado en contra de toda forma estatizante

3 El término es popularizado y estudiado por Fritz Ringer en su libro The Decline of the German Mandarins. The German Academic Community, 1890-1933. The New England University Press: EEUU, 1990. 
de la Cultura, y en tal sentido, la educación significó una preocupación para sus intereses de derribar los pilares fundacionales de occidente.

Cultura es -para Nietzsche y para la época- el paradigma formativo que gobierna en la formación humanista que pretende ofrecer la nueva Universidad; paradigma que Nietzsche coloca como una forma acrítica de transmisión de conocimientos con el objetivo de cultivar el alma del pueblo alemán. No es por azar que Nietzsche comience su ataque, en sus discursos Sobre el porvenir de nuestras instituciones escolares (1872), apuntando a la forma en la cual se produce la enseñanza: la relación entre docentes y estudiantes.

Una sola boca que habla y muchísimos oídos que escuchan y la mitad de las manos que escriben: tal es el aparato académico exterior, tal es la máquina cultural universitaria en actividad. Por lo demás, el poseedor de esta boca se encuentra separado y es independiente de los poseedores de los oídos, y esta doble autonomía es alabada con entusiasmo como libertad académica. (Nietzsche, 1995: p. 161)

¿Cuál es esa libertad académica de la que habla Nietzsche? Es simplemente una libertad aparente: la universidad es un espacio de juegos donde unos hacen que enseñan y otros hacen que aprenden, pero siempre bajo la tutela del Estado. Esta es una de las visiones más crudas de la autonomía de las universidades, en el sentido de pensar que esta autonomía es un buen engaño que la clase académica (profesores y estudiantes) parece hacerse constantemente. Y más aún, es un juego que siempre está siendo controlado por el Estado, que permite estas "libertades" pero con cierto límite:

pero detrás de estos dos grupos, a respetuosa distancia y con una cierta actitud de interventor ansioso, se encuentra el Estado para recordar de vez en cuando que él es el fin, el objetivo y la razón de ser de ese extraño proceder de los que hablan y de los que escuchan (Nietzsche, 1995: p. 161).

Como en el caso de su maestro Schopenhauer, la crítica nietzscheana es una crítica a la forma burocrática de la filosofía en las universidades. El ceremonial de la formación humanista no es más que un trámite educativo que deja, en el fondo, a estudiantes sin verdadera capacidad filosófica. Para medir esta formación se debería, primeramente, alentar la "necesidad de filosofía" que ese estudiante lleva; segundo, indagar sobre sus "instintos artísticos"; finalmente, conocer sobre "el estudio de la antigüedad griega y romana, que es el verdadero imperativo categórico concreto de toda cultura". Pero sobre todo, la crítica al sistema de educación está en la confirmación más vital y existencialista de lo que significa ser estudiante: ¿cómo es que en el momento más conflictivo de la existencia, donde las dudas y la curiosidad encuentran su máxima expresión, sometemos a los jóvenes a la máquina estatal de educación que sofoca toda pulsión de vida y le impone una racionalidad que se pretende realidad?". Más aún, Nietzsche logra ver cómo el pensamiento racional se manifiesta en las disciplinas que van a fundar el pensamiento universitario - "refugiado en el seno de la evidencia"-, en lo que dio por llamarse la "cultura histórica".

Un sistema, que hasta hace poco tiempo gozaba de una escandalosa fama mundial, ha descubierto la fórmula de esta autodestrucción de la filosofía, y hoy doquiera, según la consideración histórica de las cosas, revela hasta tal punto una ingenua ausencia de escrúpulos para transformar aquello que es lo más irracional en la razón (...) [Nietzsche, 1995: p. 164]

\section{Sobre La Naturaleza De La Autonomía}

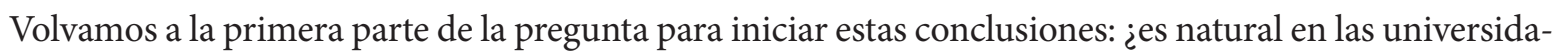
des esa situación de aislamiento? En un sentido estricto, parece obvio que una condición como la autonomía, aplicada a una institución, no está implícita en su propia existencia. Declararse autónomo, en este caso, parece

4 La crítica nietzscheana de la universidad, como la de Schopenhauer, no puede ser entendida cabalmente sin considerar la presencia de Hegel en el escenario universitario alemán de comienzos del siglo XIX. En el escrito que se está analizando,

Nietzsche vuelve a la máxima de Hegel que reza "lo real es lo racional". 
ser, antes que nada, protegerse de las injerencias de otros actores o instituciones que quedan por fuera. En cierto sentido, obedeciendo a una vieja concepción sobre el filosofar, podría decirse que la existencia de las universidades se comprende en tanto que precisamos alejarnos del mundo, de su cotidianidad, y refugiarnos en espacios creados donde se habilite, libremente, la actividad contemplativa. Como esa reconocida expresión de Paul Valéry, "tantôt je pense et tantôt je suis", donde los verbos "pensar" y "ser" parecen excluirse con necesidad; así la necesidad de la universidad emerge de la construcción de un pensar que precisa escindirse del mundo.

Peter Sloterdijk, en una serie de conferencias que dictó en la Universidad de Tübingen, Alemania, en el año 2009, sustenta la idea de la academia como un espacio heterotópico ${ }^{6}$ en las sociedades, y otorga a la creación platónica de la Academia el gesto innovador donde se funda el aislamiento de las universidades por el pensamiento.

Se propuso entonces recoger bajo una cubierta apropiada la precaria circunstancia de la dedicación total a los pensamientos. No otra cosa es la Academia originaria, considerada una innovación creadora de espacio: representa una nueva institución, sin precedentes modélicos, para albergar ausencias que se producen la búsqueda de la conexión, aún ampliamente desconocida, entre ideas; y, por qué no, en el estudio de la conexión entre palabras y cosas, que, si se considera correctamente, solo puede ser problemática. [Sloterdijk, 2013: p. 52]

Tomemos la figura griega que representa el filosofar, aquella que fue ampliamente difundida por Jenofonte, esa que describe a un Sócrates que en ocasiones era encontrado totalmente inmerso en sus pensamientos, abstraído del mundo, separado del tiempo. De esa imagen del pensamiento abstraído en la figura de Sócrates, que hicieron sus contemporáneos y la propia Historia de la Filosofía, surge el proyecto de provocar una institución que funcione bajo reglas que habiliten el libre-pensar. ¿Qué es la Universidad sino aquella institución donde se suspenden las vicisitudes cotidianas, donde el pensamiento marca su propio ritmo y el espíritu contemplativo se dirige a sí mismo?

Y será el juicio político que recibe Sócrates en la arena pública ateniense donde se funde la justificación final de toda academia. El precio de pensar en el mundo, de dedicarse a la vida contemplativa (theoria), especulativa, reflexiva en medio de los problemas del mundo, tienen como consecuencia la muerte del pensador. Esta es una clara lección que logró rescatar Platón: la filosofía no puede seguir siendo realizada en los recintos privados o en los espacios públicos, porque allí las reglas del pensamiento no son las que imperan. Es necesario, pues, elaborar un recinto para el pensamiento, para la filosofía, donde el pensar solo pueda ser juzgado por las reglas propias de la filosofía. Nace así, en este gesto aislacionista, el principio de autonomía que toda institución académica reclama.

Por lo que respecta a las consecuencias sociales de esta secesión espiritual, se manifiestan en la dramática constatación de que toda sociedad altamente desarrollada ha de contar con la existencia de contrasociedades de pensadores. Desde hace más de dos milenios y medio, una parte pequeña, pero no inesencial, de la población de nuestro hemisferio está siempre con el pensamiento en otra parte. Edificios de academias, escuelas, monasterios, iglesias y recintos de soledad muestran cómo ese en-otra-parte se articula arquitectónicamente. [Sloterdijk, 2013: p. 48]

Lo que no parece haber estado presente en los cálculos del proyecto académico griego es que, con la conformación y profesionalización de las universidades, el peligro de que una nueva clase social aumenta en posibilidades. Y esto es algo que rescatan los autores mencionados en el siglo que vio el mayor proceso de profesionalización académica. Sean los gentlemen para Newman, los mandarines alemanes, o la propia

$5 \quad$ Este "a veces pienso y a veces soy", es particularmente analizado en un texto poco referido de Hannah Arendt, ¿Dónde estamos cuando pensamos? [1984]. Allí se ubica la pregunta por el Yo pensante y su relación con el mundo, reconociendo la filósofa alemana una deuda que contraemos con Aristóteles en su fina diferenciación de la vida política y la vida filosófica.

6 Este término es retomado por Sloterdijk del pensamiento de Foucault. En forma resumida, el espacio heterotópico es aquel que se conforma entre lo utópico y lo distópico, y que "aun siendo cierto que se incluye en el entorno normal u «ortotópico» de la polis, está sujeto a sus propias leyes, chocantes, incluso incomprensibles, para la ciudad” [Sloterdijk: 2913, pag 53], La Heterotopía obedece al discurso médico, y refiere a la formación de tejidos en lugares del organismo donde normalmente no suelen formarse. Esta analogía trazada por Sloterdijk, quiere demostrar el carácter ambiguo de las universidades, que estando en la ciudad, no obedecen a sus reglas. 
"filosofía de cátedra" que denunciaron Schopenhauer o Nietzsche, lo que queda claro es que con un proyecto autónomo de universidad, se constituye un nuevo sector de la sociedad que -para sostener su condiciónalienta el juego aislacionista de la educación superior. Los rehenes de tal situación no son más que la propia Filosofía y la Ciencia.

Si el conocimiento requiere espacios especiales para su creación y cultivo, es decir, requiere de universidades; no menos cierto es que el costo de ello es permitir el surgimiento de una clase que -creando y defendiendo sus propias reglas- se mantenga aislada de su sociedad. La práctica de la ciencia moderna, la Filosofía de la Naturaleza, debe entenderse, también, como un justificativo social para el funcionamiento autónomo de las universidades, tradición que se sostiene inalterada desde la época griega. Esta situación de aislamiento, durante el nacimiento de la ciencia moderna, se ve caracterizada y reafirmada por una nueva condición: la relativa especialización que los saberes comienzan a requerir dentro del campo global del conocimiento. Es decir, un nuevo elemento confirma la necesidad de mantener a la institución académica en su condición autónoma: el mundo debe estudiarse especializadamente, y esto solo es posible si logramos forjar conocimiento especializado que permita abocarse a los eruditos en tareas estrictamente cognitivas.

Esta discusión sobre el espacio de la ciencia y la filosofía, desnuda parte de los supuestos que este trabajo ha querido mostrar. Por un lado, que si estas prácticas humanas -la filosofía, la ciencia- se han formado y desarrollado en instituciones autónomas y aisladas del resto de las dimensiones sociales, esto no significa que algo intrínseco a su práctica requiera de estas condiciones de crecimiento y desarrollo. Más bien, como se ha intentado mostrar para el caso de la Universidad, son las personas (y sus valores) y clases sociales (y sus valores) los que han impulsado una práctica aislacionista al desarrollar la filosofía, cuyo sustento no fue otro que la condición de clase, el status social o las motivaciones ideológicas.

El conocimiento no requiere per se de condiciones autónomas porque no se define por sus resultados. Es decir, lo que invitamos a pensar aquí es que no es a partir de los resultados que produce lo que definimos como conocimiento; sino por las prácticas que permiten definir los campos disciplinares, y las reglas que allí se van definiendo. Definir la empresa científica a partir del tipo especial de conocimiento que esa empresa produce, ha sido uno de los relatos más fuertes que se han constituido en la cultura occidental. El interés de este trabajo es mostrar que ciencia y filosofía, en tanto tal, se definen por esos elementos que las han ido constituyendo en las diferentes sociedades (aquí simplemente se ha presentado el caso alemán en el siglo XIX), como la clase que las promueve y la distribución de poderes que esa práctica derrama en cada contexto.

Ciencia y Filosofía ha sido, ante todo, prácticas que distribuyen poder en un mapa social, y esto, creo, puede observarse si vemos los textos de los autores presentados. El conocimiento que producen las universidades implica que sea concebido como un bien posicional, un bien que logra diferenciar a quien lo posee de quien no. Si el conocimiento se asume, ante todo, como un bien posicional, una nueva y más compleja tarea puede enunciarse para la Epistemología. Trazar los fundamentos del conocimiento no puede meramente implicar el reconocimiento de la ruta cognitiva de los individuos (conocimiento acumulativo), sino que ya no es posible desligar de este estudio dimensiones históricas, éticas, económicas, políticas e ideológicas. 
Juan Andrés Queijo Olano

\section{Bibliografía}

ARENDT, Hannah (1984) La vida del espíritu. El pensar, la voluntad y el juicio en la filosofía y en la política. Centro de Estudios Constitucionales: Madrid.

BONEVECCHIO, Claudio (1995) El mito de la Universidad. Siglo XXI Editores: Madrid.

FULLER, Steve (2009) The sociology of intellectual life. The career of the mind in and around the academy. SAGE Publications Ltd: Londres.

GILSON, Étienne (1989). La Filosofía en la Edad Media. Desde los orígenes patrióticos hasta el siglo XIV. Editorial Gredos: Madrid.

GORDON, Graham. (2005) The Institution of Intellectual Values. Realism and Idealism in Higher Education. Imprint academic: Exeter, UK.

GRANT, Edward (1996) The Foundations of Modern Science in the Middle Ages. Their religious, institutional and intellectual contexts. Cambridge University Press: U.K.

HOBSBAWN, Eric J. (2011) La era de las revoluciones. 1789 - 1848. Crítica: Buenos Aires.

KANT, Immanuel (2004) El conflicto de las Facultades. Losada: Buenos Aires.

MARTI MARCO, María Rosario (2012). Wilhelm Von Humboldt y la Creación del Sistema Universitario Moderno. Editorial Verbum: Madrid.

NIETZSCHE, Friedrich (1995) La "libre" cultura universitaria en BONEVECCHIO, Claudio, El mito de la Universidad. Siglo XXI Editores: Madrid.

NEWMAN, John Henry (2016). La idea de una Universidad. Ediciones Universidad Católica de Chile: Santiago de Chile.

RINGER, Fritz (1990). The Decline of the German Mandarins. The German Academic Community, 1890-1933. The New England University Press: EEUU.

(2000). Education, Economy, and Society in Germany, 1800-1960. En Toward a Social History of Knowledge. Bergham Books: EEUU.

SAFRANSKI, Rüdiger (2013) Schopenhauer y los años salvajes de la filosofía. Tusquets Editores: Buenos Aires.

SCHOPENHAUER, Arthur (2009) Sobre la Filosofía de la Universidad, en Parerga y Paralipómena. Vol 1. Editorial Trotta: Madrid.

SLOTERDIJK, Peter (2013) Muerte aparente en el pensar. Sobre la filosofía y la ciencia como ejercicio. Ediciones Siruela: Madrid. 